\title{
室温下铜催化制备含羰基或酯基的喹唑啉-4(3H)-亚基类衍生物
}

\author{
谢瑞龙杨新玲秦耀果谭晓庆凌 云* \\ (中国农业大学理学院应用化学系 农业部农药化学及应用重点开放实验室 北京 100193)
}

\begin{abstract}
摘要 在室温的条件下，建立了一种简单实用的铜催化制备含酮羰基或酯基的喹唑啉-4(3H)-亚基类衍生物的新方法. 实验证明以 2-溴苯甲酰乙酸甲酯类似物及脒盐酸盐为原料, 碘化亚铜为催化剂, 磷酸钾为碱, 无添加配体的情况下, 可 成功制备一系列喹唑啉类衍生物. 其结构均经 ${ }^{1} \mathrm{H}$ NMR、 ${ }^{13} \mathrm{C}$ NMR 及 HRMS 确证.
\end{abstract}

关键词＼cjkstart铜；室温；催化；喹唑啉

\section{Copper Catalyzed Synthesis of Quinazolin-4(3H)-ylidene Derivatives Containing Carbonyl and Ester Group at Room Temperature}

\author{
Xie, Ruilong Yang, Xinling Qin, Yaoguo Tan, Xiaoqing Ling, Yun* \\ (Key Laboratory of Pesticide Chemistry and Application, Ministry of Agriculture, Department of Applied Chemistry, \\ College of Science, China Agricultural University, Beijing 100193)
}

\begin{abstract}
At room temperature, a new simple and efficient copper-catalyzed method for the synthesis of quinazolin4(3H)-ylidene derivatives containing carbonyl and ester group was developed. The targets were prepared using methyl 3-(2-bromophenyl)-3-oxopropanoate analogues and amidine hydrochlorides as the initial materials, cuprous iodide as catalyst, and potassium phosphate as base at the ligand-free condition. Their structures were confirmed by ${ }^{1} \mathrm{H} N M R,{ }^{13} \mathrm{C}$ NMR and HRMS.
\end{abstract}

Keywords copper; room temperature; catalyst; quinazoline

使用过渡金属作为催化剂构建 $\mathrm{C}-\mathrm{N}$ 键，进而制备 含氮杂环类化合物, 具有反应条件温和、产率高、选择 性好以及化合物种类丰富等特点. 此外过渡金属作为催 化剂，还具有用量少，可循环再利用等优势. 因此，利 用过渡金属催化有机新反应获取新目标化合物，日益受 到有机化学家的重视 ${ }^{[1,2]}$. 到目前为止, 大部分偶联催化 反应使用 $\mathrm{Pd}, \mathrm{Ni}$ 等重金属催化剂, 虽然这样的偶联反应 能得到较高的产率, 但是这些催化剂价格高、毒性强, 对人体有害, 同时这些反应往往伴随着有机磷配体的使 用, 阻碍了这类反应在药物合成等领域的应用 ${ }^{[3,4,5]}$. 相 比之下, 金属催化剂铜具有廉价、低毒等特点值得进一 步研究. 在探寻廉价低毒的催化剂过程中, 铜催化构建 $\mathrm{C}-\mathrm{N}$ 键制备含氮杂环反应 ${ }^{[6]}$, 尤其是室温下的反应近 年来得到了迅猛的发展 ${ }^{[7,8]}$.

喹唑啉是一个具有很好生物活性的基团 ${ }^{[9]}$, 通过适
当的修饰, 其衍生物表现出很好的抗癌 ${ }^{[10]}$ 、抗肿瘤 ${ }^{[11]}$ 、 抗病毒 ${ }^{[12]}$ 、杀虫 ${ }^{[13]}$ 、杀菌 ${ }^{[14]}$ 等医用或农用生物活性. 因 此备受药物化学家的关注, 成为新杂环药物研发的热点 之一. 合成含酮羰基或酯基喹唑啉类衍生物的方法可以 由 $N$-(2-氧基苯基)甲亚胺乙酯与丙二酸二甲酯或乙酰丙 酮在四氯化锡存在下，甲苯回流制备无取代的含羰基或 酯基喹唑啉-4(3H)-亚基类衍生物 ${ }^{[15]}$; 或是 1,3,4-塞二唑 并 $[3,2-c]$ 喹唑啉类似物在强碱叔丁醇钾作用，与丙二酸 二甲酯或乙酰丙酮反应制备含羰基或酯基-2-苯基喹唑 啉-4(3H)-亚基类衍生物 ${ }^{[16]}$. 以上方法存在着反应苛刻, 合成难度大，产率较低等不足. 为了克服以上不足，本 文设计了以 2-澳苯甲酰乙酸甲酯类似物及椫盐酸盐为 原料, 碘化亚铜为催化剂, 磷酸钾为碱, 无添加配体, 室温条件下的构建 $\mathrm{C}-\mathrm{N}$ 键的新反应，并成功制备了一 系列含酮羰基或酯基的喹唑啉-4(3H)-亚基类衍生物.

\footnotetext{
*E-mail: lyun@cau.edu.cn

Received February 8, 2014; revised April 28, 2014; published online June 3, 2014

Project supported by the National Natural Science Foundation of China (No. 21272266) and the National "Twelfth Five-Year" Plan for Science \& Technology (No. 2011BAE06B05-5).

国家自然科学基金(No. 21272266)和国家 “十二五” 科技支撑计划课题(No. 2011BAE06B05-5)资助项目.
} 


\section{1 结果与讨论}

\section{1 反应条件的优化}

我们首先以 2-溴苯甲酰乙酸甲酯、正丁脒盐酸盐为 原料作模板反应，探索了催化剂、反应溶剂、碱及原料 投料摩尔比等对反应的影响, 按照第 3.2 节所述的实验 方法进行条件优化(结果见表 1). 从表 1 我们发现, 所测 试的 6 种铜催化剂中(表 1, Entries 1 6), CuI 的反应效果 最好, 产率达到 $61 \%, \mathrm{CuO}$ 的反应效果最差, 产率仅为 $10 \%$. 随后我们探索了不同溶剂对反应的影响(表 1 , Entries $7 \sim 10$ ), 甲苯作反应溶剂时产率最高, 优于乙 醇、 $\mathrm{N}, \mathrm{N}$-二甲基甲酰胺等作为溶剂时的产率, 反应在四 氢呋喃中产率最低. 接着我们对反应的碱进行了适当的 优化(表 1, Entries 11 15), 在考察的几种碱中, 只有磷 酸钾作碱时优于碳酸钾, 产率达到 $75 \%$, 其它碱产率较 差, 其中在弱碱醋酸钠中基本不反应. 在选定最优催化 剂, 最佳溶剂及碱后, 我们尝试不加催化剂(表 1, Entry 16)或碱(表 1, Entry 17), 反应无法正常进行, 无目标物 的生成. 我们对两反应物的投料比进行了笛选(表 1 , Entries 18 19). 最终选择 1a 与 2a 的物质的量比为 1: 2 时反应最佳, 产率为 $83 \%$. 当反应暴露于空气中(表 1 , Entry 20)时, 该反应也无法进行.

\section{2 喹唑啉衍生物的合成}

模板反应篮选得到的优化条件为: 2-溴苯甲酰乙酸 甲酯类似物 $1(0.50 \mathrm{mmol})$ 、脒盐酸盐 $2(1.0 \mathrm{mmol})$ 、碘 化亚铜 $(0.05 \mathrm{mmol})$ 、磷酸钾 $(2.0 \mathrm{mmol})$ 及甲苯 $(2.0 \mathrm{~mL})$, 氞气条件下室温密闭反应 $24 \mathrm{~h}$. 为探索该反应体系的适 用范围, 我们选取了不同的反应原料进行拓展, 成功制 备了一系列含酮羰基或酯基取代喹唑啉-4(3H)-亚基类 衍生物(表 2). 由表 2 的实验数据得出: 合成的目标化合 物具有较好的产率, 最高达到 91\% (表 2, Entry 13). 研 究表明(表 2, Entries 1 5, 7 8 和 10), 当反应原料 2 相 同, 原料 1 为 2-溴苯甲酰乙酸烷基酯时反应所得产率普 遍高于原料 1 为 2-溴苯甲酰丙酮类化合物的产物; $3 \mathbf{f}$ 由 于环丙基乙脒盐酸盐位阻较大的原因, 在室温下的产率 为 $51 \%$, 适当升温至 $40{ }^{\circ} \mathrm{C}$ 反应后, 产率有明显提高, 至 $81 \%$ (表 2, Entry 6); 同理, 苯甲脒盐酸盐的位阻较大, 所得目标物 3i 的产率较低, 为 47\%(表 2, Entry 9); 考察 苯环上取代基不同对反应的影响(表 2, Entries 10 13), 当苯环上 $R^{1}$ 为卤素时如氯原子, 产率最佳, $R^{1}$ 为给电子 基如甲基或甲氧基时, 产率相对较差, 均不及苯环无取 代的目标化合物. 与此同时目标化合物具有很好的官能 团容忍性，如苯环上 $\mathrm{R}^{1}$ 可为烷基、烷氧基及卤素等; $\mathrm{R}^{2}$ 可为烷基, 即构建羰基类喹唑啉, $\mathrm{R}^{2}$ 为烷氧基时, 即构 建酯基类喹唑啉; $\mathrm{R}^{3}$ 可为烷基、环烷基或者苯基. $\mathrm{R}^{1}$ 、

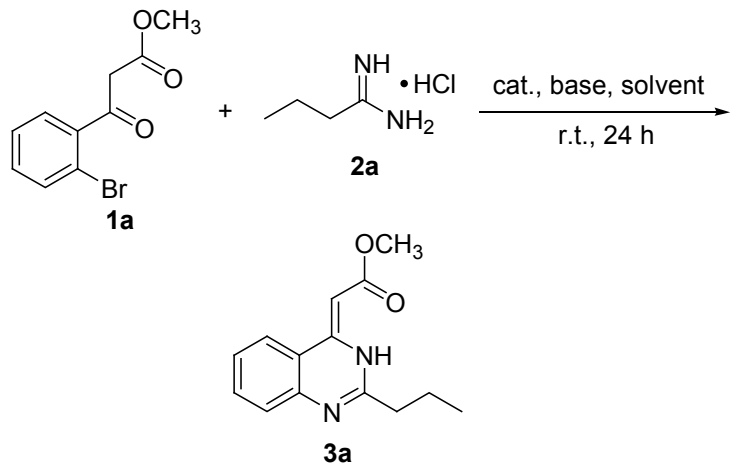

图 1 模板反应

Figure 1 Model reaction

表 1 反应条件优化 ${ }^{a}$

Table 1 Optimization of reaction conditions

\begin{tabular}{clllc}
\hline Entry & $\mathrm{Cat}$. & $\mathrm{Base}$ & Solvent $^{b}$ & Yield $^{c} \%$ \\
\hline 1 & $\mathrm{CuCl}$ & $\mathrm{K}_{2} \mathrm{CO}_{3}$ & DMF & 58 \\
2 & $\mathrm{CuBr}$ & $\mathrm{K}_{2} \mathrm{CO}_{3}$ & DMF & 49 \\
3 & $\mathrm{CuI}$ & $\mathrm{K}_{2} \mathrm{CO}_{3}$ & DMF & 61 \\
4 & $\mathrm{Cu}$ & $\mathrm{K}_{2} \mathrm{CO}_{3}$ & DMF & 52 \\
5 & $\mathrm{CuCl}$ & $\mathrm{K}_{2} \mathrm{CO}_{3}$ & DMF & 54 \\
6 & $\mathrm{CuO}$ & $\mathrm{K}_{2} \mathrm{CO}_{3}$ & DMF & 10 \\
7 & $\mathrm{CuI}$ & $\mathrm{K}_{2} \mathrm{CO}_{3}$ & DMSO & 34 \\
8 & $\mathrm{CuI}$ & $\mathrm{K}_{2} \mathrm{CO}_{3}$ & Toluene & 72 \\
9 & $\mathrm{CuI}$ & $\mathrm{K}_{2} \mathrm{CO}_{3}$ & Ethanol & 60 \\
10 & $\mathrm{CuI}$ & $\mathrm{K}_{2} \mathrm{CO}_{3}$ & THF & 15 \\
11 & $\mathrm{CuI}$ & $\mathrm{NaOAc}$ & Toluene & - \\
12 & $\mathrm{CuI}$ & $\mathrm{Na}_{2} \mathrm{CO}_{3}$ & Toluene & 21 \\
13 & $\mathrm{CuI}$ & $\mathrm{Cs}_{2} \mathrm{CO}_{3}$ & Toluene & 48 \\
14 & $\mathrm{CuI}$ & $\mathrm{K}_{3} \mathrm{PO}_{4}$ & Toluene & 75 \\
15 & $\mathrm{CuI}$ & $\mathrm{KOH}_{3}$ & Toluene & 16 \\
16 & - & $\mathrm{K}_{3} \mathrm{PO}_{4}$ & Toluene & - \\
17 & $\mathrm{CuI}$ & - & Toluene & - \\
$18^{d}$ & $\mathrm{CuI}$ & $\mathrm{K}_{3} \mathrm{PO}_{4}$ & Toluene & 57 \\
$19^{e}$ & $\mathrm{CuI}$ & $\mathrm{K}_{3} \mathrm{PO}_{4}$ & Toluene & 83 \\
$20^{f}$ & $\mathrm{CuI}$ & $\mathrm{K}_{3} \mathrm{PO}_{4}$ & Toluene & - \\
\hline
\end{tabular}

${ }^{a}$ Reaction condition: methyl 3-(2-bromophenyl)-3-oxopropanoate (1a) (0.50 $\mathrm{mmol})$, butyramidine hydrochloride $(2 \mathrm{a})(0.75 \mathrm{mmol})$, base $(2.0 \mathrm{mmol})$, solvent $(2.0 \mathrm{~mL})$ at room temperature $\left(\sim 25{ }^{\circ} \mathrm{C}\right)$ under argon atmosphere; ${ }^{b}$ DMF: $N, N$-dimethylformamide, DMSO: dimethylsulfoxide, THF: tetrahydrofuran; ${ }^{c}$ Isolated yield; ${ }^{d} \mathbf{2 a}(0.50 \mathrm{mmol}) ;{ }^{e} \mathbf{2 a}(1.00 \mathrm{mmol}) ;{ }^{f}$ Under air.

$\mathrm{R}^{2}$ 及 $\mathrm{R}^{3}$ 的不同很好的丰富了化合物的结构多样性.

由于目标产物结构新颖, 存在 $E$ 构型或者 $Z$ 构型, 我们通过对目标化合物 $3 \mathbf{i}$ 进行单晶 $X$ 射线衍射，确定了 构型为 $Z$ 构型，同时确定了活泼氢的位置. 其晶体结构 见图 2, 晶体结构数据存在英国剑桥晶体结构数据中心, CCDC 号为 1000475 .

\section{2 结论}

建立了一种以 2-溴苯甲酰乙酸甲酯类似物及㜆盐 酸盐为原料, 碘化亚铜为催化剂, 磷酸钾为碱, 室温且 无配体存在的条件下制备含酮羰基或酯基的喹唑啉- 
表 2 喹唑啉类衍生物的合成 ${ }^{a}$

Table 2 The synthesis of quinazoline derivatives

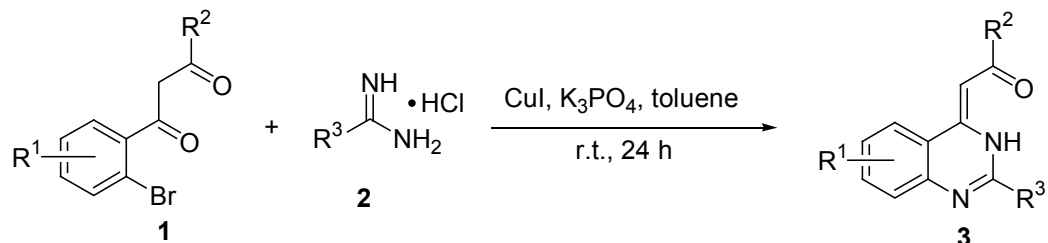

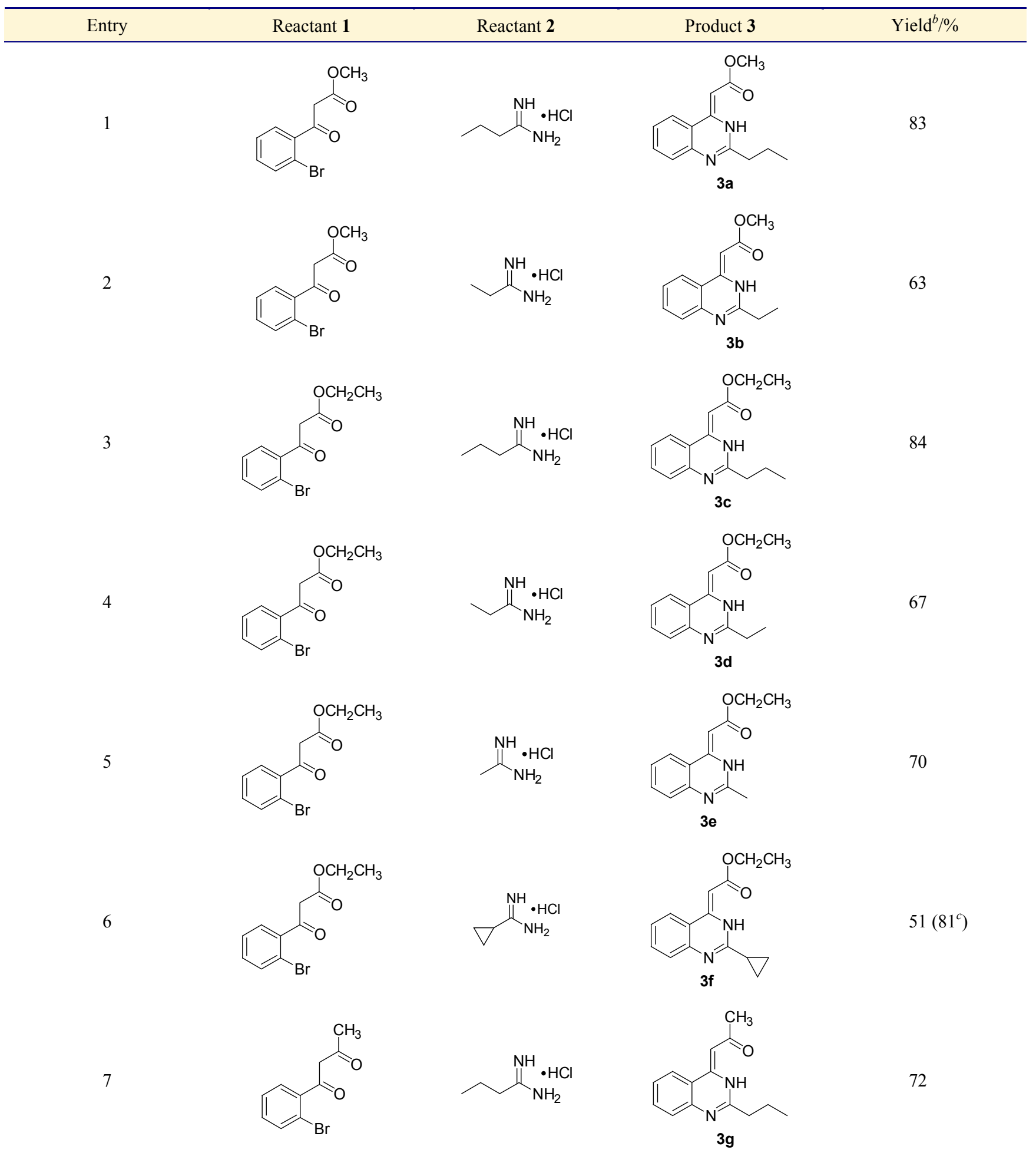




\begin{tabular}{|c|c|c|c|c|}
\hline Entry & Reactant 1 & Reactant 2 & Product 3 & Yield $^{b} / \%$ \\
\hline 8 & & & & 60 \\
\hline 9 & & & & 47 \\
\hline 10 & & & & 68 \\
\hline 11 & & & & 74 \\
\hline 12 & & & & 55 \\
\hline 13 & & & & 77 \\
\hline 14 & & & & 91 \\
\hline
\end{tabular}

${ }^{a}$ Reaction condition: methyl 3-(2-bromophenyl)-3-oxopropanoate $(\mathbf{1 a})(0.50 \mathrm{mmol})$, butyramidine hydrochloride (2a) $(0.75 \mathrm{mmol})$, base $(2.0 \mathrm{mmol})$, solvent $(2.0$ $\mathrm{mL})$ at room temperature $\left(\approx 25{ }^{\circ} \mathrm{C}\right)$ under argon atmosphere; ${ }^{b}$ isolated yield; ${ }^{c}$ at $40{ }^{\circ} \mathrm{C}$.

4(3H)-亚基类化合物的新方法. 在此催化体系中无需添 加配体, 在室温条件下就可获得较好的产率, 减少了能 源的浪费, 符合绿色化学的要求; 与此同时构建的喹唑 啉类化合物具有羰基或酯基官能团, 为化合物的进一步 衍生化提供了理想的活性位点, 在新医药或农药的创制
中有着很重要的意义.

\section{3 实验部分}

\section{1 仪器与试剂}

Cole-Parmer 熔点仪(温度计未校正); JEOL JNM- 


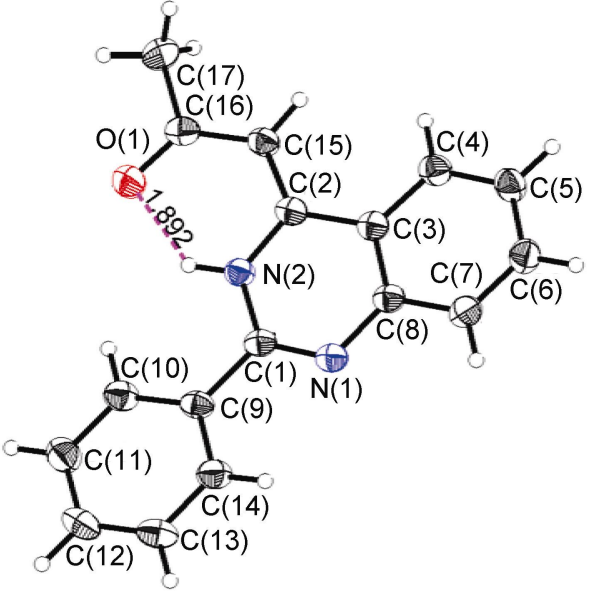

图 2 目标化合物 $3 \mathbf{i}$ 的晶体结构图

Figure 2 Crystal structure of the target compound $\mathbf{3 i}$

ECA $300 \mathrm{MHz}$, JEOL JNM-ECA $400 \mathrm{MHz}$ 及 JEOL JNM-ECA $600 \mathrm{MHz}$ 核磁共振仪(以 $\mathrm{TMS}$ 为内标, $\mathrm{CDCl}_{3}$ 及 DMSO- $d_{6}$ 为溶剂); APEX IV 傅立叶变换高分辨质谱; RAPID-S 影像板 X 射线(单晶)衍射仪. 起始原料2-溴苯 甲酰乙酸甲酯类似物 1 参照文献 $[17,18]$ 制备. 所用试剂 均为市售分析纯或化学纯试剂, 溶剂用前均经无水处 理.

\section{2 实验方法}

合成步骤: 在 $25 \mathrm{~mL}$ 反应管中依次加入 2-溴苯甲酰 乙酸甲酯类似物 $\mathbf{1}(0.50 \mathrm{mmol}) 、$ 脒盐酸盐 2 (1.0 mmol), 碘化亚铜 $(0.05 \mathrm{mmol}) 、$ 磷酸钾 $(2.0 \mathrm{mmol})$ 及甲苯 $(2.0 \mathrm{~mL})$, 氩气条件下密闭反应. 室温下反应 $24 \mathrm{~h}$ 后, 将反应液倒 入 $15 \mathrm{~mL}$ 水中, 用乙酸乙酯萃取 3 次, 每次 $30 \mathrm{~mL}$, 合并 有机相, 无水硫酸钠干燥 $0.5 \mathrm{~h}$ 后, 旋干得到粗产物. 柱 层析 $[V$ (乙酸乙酯 $): V($ 正己烷 $)=1: 5]$ 纯化得到目标化 合物 3. 计算产率并通过 ${ }^{1} \mathrm{H} \mathrm{NMR},{ }^{13} \mathrm{C} \mathrm{NMR}$ 及高分辨质 谱进行结构表征.

单晶培养: $30 \mathrm{mg}$ 目标物 $3 \mathbf{i}$ 溶解在含有 $5 \mathrm{~mL}$ 丙酮溶 剂的单口三角瓶中, 溶液略带黄色. 用封口膜封口, 但 留有少许间隙. 静置于冰箱冷藏室(约 $4{ }^{\circ} \mathrm{C}$ ), 待溶剂缓 慢挥发直至析出得到单晶样品.

\section{3 目标物结构表征}

(Z)-2'-[2-丙基喹唑啉-4(3H)- 亚基] 乙酸甲酯(3a): $101 \mathrm{mg}$ 无色粘稠状液体, 产率 $83 \%$. ${ }^{1} \mathrm{H} \mathrm{NMR}\left(\mathrm{CDCl}_{3}\right.$, $300 \mathrm{MHz}) \delta$ : 12.35 (brs, $1 \mathrm{H}), 7.72$ (d, $J=7.91 \mathrm{~Hz}, 1 \mathrm{H})$, $7.58 \sim 7.49(\mathrm{~m}, 2 \mathrm{H}), 7.30(\mathrm{t}, J=6.88 \mathrm{~Hz}, 1 \mathrm{H}), 5.49(\mathrm{~s}, 1 \mathrm{H})$, $3.76(\mathrm{~s}, 3 \mathrm{H}), 2.63(\mathrm{t}, J=7.91 \mathrm{~Hz}, 2 \mathrm{H}), 1.93 \sim 1.80(\mathrm{~m}, 2 \mathrm{H})$, $1.06(\mathrm{t}, J=7.23 \mathrm{~Hz}, 3 \mathrm{H}) ;{ }^{13} \mathrm{C} \mathrm{NMR}\left(\mathrm{CDCl}_{3}, 75 \mathrm{MHz}\right) \delta$ : $171.4,154.7,150.4,145.2,133.1,127.6,126.3,123.1$, 118.8, 78.0, 50.9, 38.2, 20.8, 13.8; HRMS calcd for
$\mathrm{C}_{14} \mathrm{H}_{17} \mathrm{~N}_{2} \mathrm{O}_{2}(\mathrm{M}+\mathrm{H})^{+}$245.1285, found 245.1284.

(Z)-2'-[2-乙基喹唑啉-4(3H)-亚基]乙酸甲酯(3b)：72 $\mathrm{mg}$ 白色固体, 产率 $63 \%$. m.p. $77 \sim 78{ }^{\circ} \mathrm{C} ;{ }^{1} \mathrm{H} \mathrm{NMR}$ $\left(\mathrm{CDCl}_{3}, 600 \mathrm{MHz}\right) \delta: 12.38$ (brs, $\left.1 \mathrm{H}\right), 7.71$ (d, $J=8.25 \mathrm{~Hz}$, $1 \mathrm{H}), 7.57$ (t, $J=6.87 \mathrm{~Hz}, 1 \mathrm{H}), 7.50$ (d, $J=8.25 \mathrm{~Hz}, 1 \mathrm{H})$, $7.30(\mathrm{t}, J=7.56 \mathrm{~Hz}, 1 \mathrm{H}), 5.48(\mathrm{~s}, 1 \mathrm{H}), 3.76(\mathrm{~s}, 3 \mathrm{H}), 2.69$ $(\mathrm{q}, J=7.56 \mathrm{~Hz}, 2 \mathrm{H}), 1.40(\mathrm{t}, J=8.25 \mathrm{~Hz}, 3 \mathrm{H}) ;{ }^{13} \mathrm{C} \mathrm{NMR}$ $\left(\mathrm{CDCl}_{3}, 150 \mathrm{MHz}\right) \delta: 171.4,155.5,150.4,145.4,133.1$, 127.6, 126.3, 123.1, 118.9, 78.1, 50.9, 29.4, 11.2; HRMS calcd for $\mathrm{C}_{13} \mathrm{H}_{15} \mathrm{~N}_{2} \mathrm{O}_{2}(\mathrm{M}+\mathrm{H})^{+} 231.1128$, found 231.1129 .

(Z)-2'-[2-丙基喹唑啉-4(3H)-亚基]乙酸乙酯(3c): 109 $\mathrm{mg}$ 无色粘稠状液体, 产率 84\%. ${ }^{1} \mathrm{H}$ NMR ( $d_{6}$-DMSO, $600 \mathrm{MHz}) \delta: 12.29$ (brs, $1 \mathrm{H}), 8.01$ (d, $J=8.25 \mathrm{~Hz}, 1 \mathrm{H})$, $7.64(\mathrm{t}, J=6.87 \mathrm{~Hz}, 1 \mathrm{H}), 7.44(\mathrm{~d}, J=7.56 \mathrm{~Hz}, 1 \mathrm{H}), 7.36(\mathrm{t}$, $J=7.56 \mathrm{~Hz}, 1 \mathrm{H}), 5.63(\mathrm{~s}, 1 \mathrm{H}), 4.15(\mathrm{q}, J=6.87 \mathrm{~Hz}, 2 \mathrm{H})$, $2.63(\mathrm{t}, J=7.56 \mathrm{~Hz}, 2 \mathrm{H}), 1.79 \sim 1.73(\mathrm{~m}, 2 \mathrm{H}), 1.25(\mathrm{t}, J=$ $6.87 \mathrm{~Hz}, 3 \mathrm{H}), 0.98(\mathrm{t}, J=6.87 \mathrm{~Hz}, 3 \mathrm{H}) ;{ }^{13} \mathrm{C}$ NMR $\left(d_{6}\right.$-DMSO, $\left.150 \mathrm{MHz}\right) \delta: 170.7,154.6,150.2,145.4,133.8$, $127.6,127.0,124.2,118.8,78.4,59.5,37.5,20.2,14.9$, 14.0; HRMS calcd for $\mathrm{C}_{15} \mathrm{H}_{19} \mathrm{~N}_{2} \mathrm{O}_{2}(\mathrm{M}+\mathrm{H})^{+}$259.1441, found 259.1439 .

(Z)-2'-[2-乙基喹唑啉-4(3H)-亚基]乙酸乙酯(3d)：82 $\mathrm{mg}$ 白色固体，产率 67\%. m.p. 56 57 ${ }^{\circ} \mathrm{C} ;{ }^{1} \mathrm{H} \mathrm{NMR}$ $\left(\mathrm{CDCl}_{3}, 600 \mathrm{MHz}\right) \delta: 12.41$ (brs, $\left.1 \mathrm{H}\right), 7.72$ (d, $J=6.87 \mathrm{~Hz}$, $1 \mathrm{H}), 7.57$ (t, $J=6.87 \mathrm{~Hz}, 1 \mathrm{H}), 7.50$ (d, $J=7.56 \mathrm{~Hz}, 1 \mathrm{H})$, $7.28(\mathrm{t}, J=6.87 \mathrm{~Hz}, 1 \mathrm{H}), 5.48(\mathrm{~s}, 1 \mathrm{H}), 4.22(\mathrm{q}, J=6.87 \mathrm{~Hz}$, 2H), 2.69 (q, $J=7.56 \mathrm{~Hz}, 2 \mathrm{H}), 1.40$ (t, $J=7.56 \mathrm{~Hz}, 3 \mathrm{H})$, $1.33(\mathrm{t}, J=6.87 \mathrm{~Hz}, 3 \mathrm{H}) ;{ }^{13} \mathrm{C} \mathrm{NMR}\left(\mathrm{CDCl}_{3}, 150 \mathrm{MHz}\right) \delta$ : $171.1,155.6,150.3,145.4,133.0,127.6,126.3,123.1$, $118.9,78.5,59.5,29.4,14.6,11.3$; HRMS calcd for $\mathrm{C}_{14} \mathrm{H}_{17} \mathrm{~N}_{2} \mathrm{O}_{2}(\mathrm{M}+\mathrm{H})^{+}$245.1284, found 245.1285.

(Z)-2'-[2-甲基喹唑啉-4(3H)-亚基]乙酸乙酯(3e)：80 $\mathrm{mg}$ 白色固体，产率 $70 \%$. m.p. $64 \sim 65{ }^{\circ} \mathrm{C} ;{ }^{1} \mathrm{H} \mathrm{NMR}$ $\left(\mathrm{CDCl}_{3}, 600 \mathrm{MHz}\right) \delta: 12.30$ (brs, $\left.1 \mathrm{H}\right), 7.70$ (d, $J=8.25 \mathrm{~Hz}$, $1 \mathrm{H}), 7.56$ (t, $J=6.87 \mathrm{~Hz}, 1 \mathrm{H}), 7.47$ (d, $J=8.25 \mathrm{~Hz}, 1 \mathrm{H})$, $7.30 \sim 7.27(\mathrm{~m}, 1 \mathrm{H}), 5.46(\mathrm{~s}, 1 \mathrm{H}), 4.21(\mathrm{q}, J=6.87 \mathrm{~Hz}$, $2 \mathrm{H}), 2.41(\mathrm{~s}, 3 \mathrm{H}), 1.33(\mathrm{t}, J=7.56 \mathrm{~Hz}, 3 \mathrm{H}) ;{ }^{13} \mathrm{C}$ NMR $\left(\mathrm{CDCl}_{3}, 150 \mathrm{MHz}\right) \delta: 171.0,151.2,150.1,145.2,133.0$, 127.4, 126.3, 123.0, 118.7, 78.6, 59.5, 22.6, 14.6; HRMS calcd for $\mathrm{C}_{13} \mathrm{H}_{15} \mathrm{~N}_{2} \mathrm{O}_{2}(\mathrm{M}+\mathrm{H})^{+}$231.1128, found 231.1128 .

(Z)-2'-[2-环丙基喹唑啉-4(3H)-亚基]乙酸乙酯(3f): $104 \mathrm{mg}$ 白色固体, 产率 $81 \%$. m.p. 74 76 ${ }^{\circ} \mathrm{C}$; ${ }^{1} \mathrm{H} \mathrm{NMR}$ $\left(\mathrm{CDCl}_{3}, 300 \mathrm{MHz}\right) \delta: 12.38$ (brs, $\left.1 \mathrm{H}\right), 7.64$ (d, $J=8.26 \mathrm{~Hz}$, $1 \mathrm{H}), 7.49$ (t, $J=8.26 \mathrm{~Hz}, 1 \mathrm{H}), 7.40$ (d, $J=6.88 \mathrm{~Hz}, 1 \mathrm{H})$, 
7.19 (t, $J=8.26 \mathrm{~Hz}, 1 \mathrm{H}), 5.40$ (s, 1H), $4.18(\mathrm{q}, J=7.22 \mathrm{~Hz}$, $2 \mathrm{H}), 1.83 \sim 1.74(\mathrm{~m}, 1 \mathrm{H}), 1.30(\mathrm{t}, J=6.88 \mathrm{~Hz}, 3 \mathrm{H}), 1.21 \sim$ $1.16(\mathrm{~m}, 2 \mathrm{H}), 1.05 \sim 0.98(\mathrm{~m}, 2 \mathrm{H}) ;{ }^{13} \mathrm{C} \mathrm{NMR}\left(\mathrm{CDCl}_{3}, 75\right.$ MHz) $\delta: 171.1,155.7,150.3,145.7,132.9,127.4,125.7$, 123.1, 118.8, 78.0, 59.4, 15.4, 14.6, 8.6; HRMS calcd for $\mathrm{C}_{15} \mathrm{H}_{17} \mathrm{~N}_{2} \mathrm{O}_{2}(\mathrm{M}+\mathrm{H})^{+}$257.1285, found 257.1286.

(Z)-1'-[2-丙基喹唑啉-4(3H)-亚基]丙-2-酮(3g)：82 $\mathrm{mg}$ 黄色固体, 产率 $72 \%$. m.p. $124 \sim 125{ }^{\circ} \mathrm{C} ;{ }^{1} \mathrm{H}$ NMR $\left(\mathrm{CDCl}_{3}, 300 \mathrm{MHz}\right) \delta$ : 14.67 (brs, $\left.1 \mathrm{H}\right), 7.83(\mathrm{~d}, J=8.54 \mathrm{~Hz}$, 1H), 7.66 (t, $J=7.93 \mathrm{~Hz}, 1 \mathrm{H}), 7.58$ (d, $J=7.93 \mathrm{~Hz}, 1 \mathrm{H})$, $7.38(\mathrm{t}, J=8.54 \mathrm{~Hz}, 1 \mathrm{H}), 6.02(\mathrm{~s}, 1 \mathrm{H}), 2.70(\mathrm{t}, J=7.93 \mathrm{~Hz}$, $2 \mathrm{H}), 2.25(\mathrm{~s}, 3 \mathrm{H}), 1.94 \sim 1.85(\mathrm{~m}, 2 \mathrm{H}), 1.05(\mathrm{t}, J=7.32 \mathrm{~Hz}$, $3 \mathrm{H}) ;{ }^{13} \mathrm{C}$ NMR $\left(\mathrm{CDCl}_{3}, 75 \mathrm{MHz}\right) \delta: 197.0,155.0,151.2$, 146.5, 133.6, 127.9, 126.6, 123.3, 118.1, 88.9, 38.2, 29.9, 20.7, 13.8; HRMS calcd for $\mathrm{C}_{14} \mathrm{H}_{17} \mathrm{~N}_{2} \mathrm{O}(\mathrm{M}+\mathrm{H})^{+}$ 229.1335 , found 229.1336 .

(Z)-1'-[2-乙基喹唑啉-4(3H)-亚基]丙-2-酮(3h)：64 $\mathrm{mg}$ 黄色固体, 产率 $60 \%$. m.p. $104 \sim 106{ }^{\circ} \mathrm{C} ;{ }^{1} \mathrm{H}$ NMR $\left(\mathrm{CDCl}_{3}, 400 \mathrm{MHz}\right) \delta: 14.67$ (brs, $\left.1 \mathrm{H}\right), 7.83$ (d, $J=8.54 \mathrm{~Hz}$, 1H), 7.66 (t, $J=7.93 \mathrm{~Hz}, 1 \mathrm{H}), 7.59$ (d, $J=7.92 \mathrm{~Hz}, 1 \mathrm{H})$, $7.38(\mathrm{t}, J=7.32 \mathrm{~Hz}, 1 \mathrm{H}), 6.02(\mathrm{~s}, 1 \mathrm{H}), 2.77$ (q, $J=7.93 \mathrm{~Hz}$, $2 \mathrm{H}), 2.26$ (s, 3H), 1.43 (t, $J=7.93 \mathrm{~Hz}, 3 \mathrm{H}) ;{ }^{13} \mathrm{C} \mathrm{NMR}$ $\left(\mathrm{CDCl}_{3}, 100 \mathrm{MHz}\right) \delta$ : 197.0, 155.9, 151.2, 146.6, 133.6, 127.8, 126.6, 123.3, 118.1, 88.9, 29.9, 29.5, 11.3; HRMS calcd for $\mathrm{C}_{13} \mathrm{H}_{15} \mathrm{~N}_{2} \mathrm{O}(\mathrm{M}+\mathrm{H})^{+} 215.1179$, found 215.1178.

(Z)-1'-[2-苯基喹唑啉-4(3H)-亚基]丙-2-酮(3i): 62 $\mathrm{mg}$ 黄色固体, 产率 47\%. m.p. $141 \sim 142{ }^{\circ} \mathrm{C}$ (lit. ${ }^{[16]}$ m.p. 138 $\left.140{ }^{\circ} \mathrm{C}\right) ;{ }^{1} \mathrm{H} \mathrm{NMR}\left(\mathrm{CDCl}_{3}, 400 \mathrm{MHz}\right) \delta$ : 15.69 (brs, $1 \mathrm{H}), 8.27 \sim 8.25(\mathrm{~m}, 2 \mathrm{H}), 7.90(\mathrm{~d}, J=7.54 \mathrm{~Hz}, 1 \mathrm{H}), 7.76 \sim$ $7.69(\mathrm{~m}, 2 \mathrm{H}), 7.58 \sim 7.54(\mathrm{~m}, 3 \mathrm{H}), 7.42(\mathrm{td}, J=8.12 \mathrm{~Hz}$, $J=1.16 \mathrm{~Hz}, 1 \mathrm{H}), 6.12(\mathrm{~s}, 1 \mathrm{H}), 2.31$ (s, 3H); ${ }^{13} \mathrm{C} \mathrm{NMR}$ $\left(\mathrm{CDCl}_{3}, 100 \mathrm{MHz}\right) \delta: 196.7,151.8,150.2,147.0,133.8$, $132.8,131.7,129.2,128.7,127.0,126.9,123.4,118.3$, 89.1, 29.7.

(Z)-1'-[2-丙基喹唑啉-4(3H)-亚基]丁-2-酮(3j)：82 $\mathrm{mg}$ 白色固体, 产率 $68 \%$. m.p. $61 \sim 62{ }^{\circ} \mathrm{C} ;{ }^{1} \mathrm{H}$ NMR $\left(\mathrm{CDCl}_{3}, 400 \mathrm{MHz}\right) \delta$ : 14.65 (brs, $\left.1 \mathrm{H}\right), 7.83(\mathrm{~d}, J=8.12 \mathrm{~Hz}$, $1 \mathrm{H}), 7.64(\mathrm{t}, J=8.12 \mathrm{~Hz}, 1 \mathrm{H}), 7.58(\mathrm{~d}, J=7.54 \mathrm{~Hz}, 1 \mathrm{H})$, 7.36 (t, $J=8.12 \mathrm{~Hz}, 1 \mathrm{H}), 6.01$ (s, $1 \mathrm{H}), 2.70$ (t, $J=7.54 \mathrm{~Hz}$, $2 \mathrm{H}), 2.53$ (q, $J=7.54 \mathrm{~Hz}, 2 \mathrm{H}), 1.95 \sim 1.96(\mathrm{~m}, 2 \mathrm{H}), 1.21$ (t, $J=7.54 \mathrm{~Hz}, 3 \mathrm{H}), 1.06(\mathrm{t}, J=7.54 \mathrm{~Hz}, 3 \mathrm{H}) ;{ }^{13} \mathrm{C} \mathrm{NMR}$ $\left(\mathrm{CDCl}_{3}, 100 \mathrm{MHz}\right) \delta$ : 200.7, 155.0, 151.1, 146.5, 133.5, 127.8, 126.5, 123.2, 118.3, 87.9, 38.2, 35.8, 20.8, 13.8, 9.9; HRMS calcd for $\mathrm{C}_{15} \mathrm{H}_{19} \mathrm{~N}_{2} \mathrm{O}(\mathrm{M}+\mathrm{H})^{+}$243.1492, found 243.1491 .
(Z)-2'-[2-丙基-6-甲基喹唑啉-4(3H)-亚基]乙酸乙酯 (3k): $100 \mathrm{mg}$ 无色粘稠状液体, 产率 $74 \% .{ }^{1} \mathrm{H}$ NMR $\left(\mathrm{CDCl}_{3}, 400 \mathrm{MHz}\right) \delta: 12.38$ (brs, $\left.1 \mathrm{H}\right), 7.52(\mathrm{~s}, 1 \mathrm{H}), 7.40$ (s, 2H), 5.45 (s, 1H), 4.21 (q, $J=7.15 \mathrm{~Hz}, 2 \mathrm{H}), 2.62(\mathrm{t}, J=$ $7.70 \mathrm{~Hz}, 2 \mathrm{H}), 2.41(\mathrm{~s}, 3 \mathrm{H}), 1.90 \sim 1.67(\mathrm{~m}, 2 \mathrm{H}), 1.32(\mathrm{t}$, $J=7.15 \mathrm{~Hz}, 3 \mathrm{H}), 1.04(\mathrm{t}, J=7.70 \mathrm{~Hz}, 3 \mathrm{H}) ;{ }^{13} \mathrm{C} \mathrm{NMR}$ $\left(\mathrm{CDCl}_{3}, 100 \mathrm{MHz}\right) \delta: 171.2,153.9,150.3,143.3,136.2$, 134.4, 127.4, 122.9, 118.6, 78.0, 59.4, 38.2, 21.4, 20.8, 14.6, 13.8; HRMS calcd for $\mathrm{C}_{16} \mathrm{H}_{21} \mathrm{~N}_{2} \mathrm{O}_{2}(\mathrm{M}+\mathrm{H})^{+}$ 273.1598, found 273.1599 .

(Z)-2'-[2-乙基-6-甲基喹唑啉-4(3H)-亚基]乙酸乙酯 (31): $71 \mathrm{mg}$ 白色固体, 产率 55\%. m.p. $57 \sim 58{ }^{\circ} \mathrm{C} ;{ }^{1} \mathrm{H}$ NMR $\left(\mathrm{CDCl}_{3}, 300 \mathrm{MHz}\right) \delta: 12.38$ (brs, $\left.1 \mathrm{H}\right), 7.52(\mathrm{~s}, 1 \mathrm{H})$, 7.40 (s, 2H), 5.46 (s, 1H), 4.21 (q, $J=7.54 \mathrm{~Hz}, 2 \mathrm{H}), 2.68$ (q, $J=7.54 \mathrm{~Hz}, 2 \mathrm{H}), 2.40$ (s, 3H), 1.39 (t, $J=7.54 \mathrm{~Hz}, 3 \mathrm{H}$ ), $1.33(\mathrm{t}, J=6.69 \mathrm{~Hz}, 3 \mathrm{H}) ;{ }^{13} \mathrm{C} \mathrm{NMR}\left(\mathrm{CDCl}_{3}, 75 \mathrm{MHz}\right) \delta$ : $171.1,154.8,150.3,143.3,136.2,134.4,127.4,122.9$, 118.6, 78.0, 59.4, 29.4, 21.5, 14.6, 11.3; HRMS calcd for $\mathrm{C}_{15} \mathrm{H}_{19} \mathrm{~N}_{2} \mathrm{O}_{2}(\mathrm{M}+\mathrm{H})^{+}$259.1441, found 259.1441.

(Z)-2'-[2-丙基-6-甲氧基喹唑啉-4(3H)-亚基]乙酸甲 酯(3m): $105 \mathrm{mg}$ 淡黄色粘稠状液体, 产率 $77 \% .{ }^{1} \mathrm{H}$ NMR $\left(\mathrm{CDCl}_{3}, 600 \mathrm{MHz}\right) \delta$ : 12.33 (brs, $\left.1 \mathrm{H}\right), 7.42$ (d, $J=$ $8.94 \mathrm{~Hz}, 1 \mathrm{H}), 7.16(\mathrm{dd}, J=8.94,2.75 \mathrm{~Hz}, 1 \mathrm{H}), 7.02(\mathrm{~d}, J=$ $2.75 \mathrm{~Hz}, 1 \mathrm{H}), 5.35$ (s, 1H), $3.82(\mathrm{~s}, 3 \mathrm{H}), 3.73(\mathrm{~s}, 3 \mathrm{H}), 2.59$ (t, $J=7.56 \mathrm{~Hz}, 2 \mathrm{H}), 1.86 \sim 1.79(\mathrm{~m}, 2 \mathrm{H}), 1.02(\mathrm{t}, J=6.87$ $\mathrm{Hz}, 3 \mathrm{H}) ;{ }^{13} \mathrm{C} \mathrm{NMR}\left(\mathrm{CDCl}_{3}, 150 \mathrm{MHz}\right) \delta: 171.4,157.9$, $152.6,150.3,139.8,129.0,122.1,119.4,104.3,77.3,55.6$, 50.9, 38.0, 20.8, 13.8; HRMS calcd for $\mathrm{C}_{15} \mathrm{H}_{19} \mathrm{~N}_{2} \mathrm{O}_{3}$ (M+ H) ${ }^{+} 275.1390$, found 275.1390 .

(Z)-2'-[2-丙基-6-氯基喹唑啉- 4(3H)-亚基]乙酸甲 酯(3n): $126 \mathrm{mg}$ 淡黄色固体, 产率 91\%. m.p. 94 96 ${ }^{\circ} \mathrm{C}$; ${ }^{1} \mathrm{H}$ NMR $\left(\mathrm{CDCl}_{3}, 600 \mathrm{MHz}\right) \delta$ : 12.31 (brs, $\left.1 \mathrm{H}\right), 7.65$ (d, $J=2.06 \mathrm{~Hz}, 1 \mathrm{H}), 7.49(\mathrm{dd}, J=8.25,2.06 \mathrm{~Hz}, 1 \mathrm{H}), 7.42(\mathrm{~d}$, $J=8.94 \mathrm{~Hz}, 1 \mathrm{H}), 5.40(\mathrm{~s}, 1 \mathrm{H}), 3.76(\mathrm{~s}, 3 \mathrm{H}), 2.61(\mathrm{t}, J=$ $8.25 \mathrm{~Hz}, 2 \mathrm{H}), 1.89 \sim 1.82(\mathrm{~m}, 2 \mathrm{H}), 1.05(\mathrm{t}, J=7.56 \mathrm{~Hz}$, $3 \mathrm{H}) ;{ }^{13} \mathrm{C} \mathrm{NMR}\left(\mathrm{CDCl}_{3}, 150 \mathrm{MHz}\right) \delta: 171.24,155.0,149.0$, 143.2, 133.2, 131.8, 129.0, 122.7, 120.0, 79.0, 51.0, 38.1, 20.6, 13.8; HRMS calcd for $\mathrm{C}_{14} \mathrm{H}_{16} \mathrm{ClN}_{2} \mathrm{O}_{2}(\mathrm{M}+\mathrm{H})^{+}$ 279.0895, found 279.0897.

致谢 本论文得到清华大学化学系付华老师的帮助及 指导, 在此表示诚挚的谢意.

\section{References}

[1] Cheng, Y.-J.; Sun, L.-P. Chin. J. Org. Chem. 2013, 33, 877 (in Chinese). 
(成宜娟, 孙雨萍, 有机化学, 2013, 33, 877.)

[2] Rao, H.-H.; Fu, H. Synlett 2011, 745

[3] He, T.; Ge, Y.-C., Wu, L.-L.; Fu, H.-Y.; Chen, H.; Li, X.-J. Chin. J. Catal. 2011, 32, 1376 (in Chinese).

(何亭, 葛轶岑, 武垒垒, 付海燕, 陈华, 李贤均, 催化学报, 2011, 32, 1376.)

[4] Li, Z.-K.; Wu, Z.-Q.; Deng, H.; Zhou, X.-G. Chin. J. Org. Chem. 2013, 33, 760 (in Chinese).

(李正凯, 吴之清, 邓杭, 周向葛, 有机化学, 2013, 33, 760.)

[5] Xu, H.-J.; Man. Q.-S.; Lin, Y.-C.; Li. Y.-Y.; Feng, Y.-S. Chin. J. Org. Chem. 2010, 30, 9 (in Chinese).

(许华建, 蔄秋石, 林义成, 李源源, 冯乙巳, 有机化学, 2010, 30, 9.)

[6] (a) Evano, G.; Blanchard, N.; Toumi, M. Chem. Rev. 2008, 108, 3054 .

(b) Truong, V.-L.; Morrow, M. Tetrahedron Lett. 2010, 51, 758.

[7] Liu, X.-W.; Fu, H.; Jiang, Y.-Y.; Zhao, Y.-F. Angew. Chem., Int. Ed. 2009, 48, 348.

[8] Diao, X.-Q.; Wang, Y.-J.; Jiang, Y.-W.; Ma, D.-W. J. Org. Chem. 2009, 74, 7974

[9] (a) Lv, Y.-H.; Li, Y.; Xiong, T.; Pu, W.-Y.; Zhang, H.-W.; Sun, K.; Liu, Q.; Zhang, Q. Chem. Commun. 2013, 49, 6439.

(b) Gao, Y.-L.; Xiong, Q.-Z.; An, R.; Bao, X.-P. Chin. J. Org. Chem. 2011, 31, 1529 (in Chinese).
(高元否, 熊启中, 安锐, 鲍小平, 有机化学, 2011, 31, 1529.)

[10] Kim, Y.; Li, Z.-M.; Apetri, M.; Luo, B.-B.; Settleman, J.-E.; Anderson, K.-S. Biochemistry 2012, 51, 5212.

[11] Cao, S.-L.; Feng, Y.-P.; Jiang, Y.-Y.; Liu, S.-Y.; Ding, G.-Y.; Li, R.-T. Bioorg. Med. Chem. Lett. 2005, 15, 1915.

[12] Bianco, A.; Reghellin, V.; Donnici, L.; Fenu, S.; Alvarez, R.; Baruffa, C.; Peri, F.; Pagani, M.; Abrignani, S.; Neddermann, P.; De Francesco, R. PLoS Pathog. 2012, 8, e1002576.

[13] Lamberth, C.; Hillesheim, E.; Bassand, D.; Schaub, F. Pest Manage. Sci. 2000, 56, 94.

[14] (a) Ding, M.-W.; Yang, S.-J.; Chen Y.-F. Chin. J. Org. Chem. 2004, 24, 923 (in Chinese)

(丁明武，杨尚君，陈云峰，有机化学, 2004, 24, 923.)

(b) Liu, G.; Song, B.-A.; Sang, W.-J.; Yang, S.; Jin, L.-H.; Ding, X. Chin. J. Org. Chem. 2004, 24, 1296 (in Chinese).

(刘刚, 宋宝安, 桑维钧, 杨松, 金林红, 丁雄, 有机化学, 2004, 24, 1296.)

[15] Chattopadhyay, S.-K.; Dey, R.; Biswas, S. Synthesis 2005, 1083.

[16] Molina, P.; Arques, A.; Cartagena, I.; Valcarcel, M. V. J. Heterocycl. Chem. 1985, 22, 1189.

[17] Xu, H.; Li, S.-F.; Liu, H.-X.; Fu, H.; Jiang. Y.-Y. Chem. Commun. 2010, 46, 7617.

[18] Zhao, J.; Zhao, Y.-F.; Fu, H. Angew. Chem., Int. Ed. 2011, 50, 3769.

(Li, L.; Fan, Y.) 